\title{
ESTIMULACIÓN ELÉCTRICA DE CANALES DE ALPACAS PARA MEJORAR SU CALIDAD ORGANOLÉPTICA
}

\author{
Oscar Guerrero M. ${ }^{1}$, Miguel Vilca L. ${ }^{2}$, Daphne Ramos D. ${ }^{2}$, Enrique Lucho C. ${ }^{2}$ \\ y Néstor Falcón P. ${ }^{3}$
}

\section{Abstract}

Electrical stimulation is a technique that may reduce the lenght of maturity, rigor mortis and acidity of meat, as well as improving sensory characteristics. The objetive of the study was to show that the electrical stimulation has a significant effect on the quality of alpaca meat such as tenderness, juiceness, flavor, odor and sanitary condition. Twelve female alpacas from 4 to 5 year old and 50-60 kg of body weight were used. They were slaughtered and electrically stimulated 60 minutes later using electrodes on muscular tissue. All half carcasses were stimulated with $500 \mathrm{v}$ per $60 \mathrm{~s}$ (T1), $500 \mathrm{v}$ per $30 \mathrm{~s}$ (T2), $600 \mathrm{v}$ per $60 \mathrm{~s}$ (T3), and $600 \mathrm{v}$ per $30 \mathrm{~s}$ (T4). All other half carcasses were kept without electrically stimulation. The results indicated that the electrical stimulation had a significant effect $(\mathrm{p}=0.00)$ on the $\mathrm{pH}$ alpaca meat reducing microbial growth $24 \mathrm{~h}$ after stimulation $(\mathrm{p}=0.004)$, where treatment T2 $(500 \mathrm{v} / 60 \mathrm{~s})$ showed the best effect. The evaluation of sensory characteristics showed a significant effect for odor on treatment $\mathrm{T} 1(\mathrm{p}=0.009)$, flavor on treatment T4 $(\mathrm{p}=0.002)$, tenderness on treatments $\mathrm{T} 2(\mathrm{p}=0.023)$ and $\mathrm{T} 3(\mathrm{p}=0.004)$, and juiceness on treatment $\mathrm{T} 4(\mathrm{p}=0.005)$.

Key words: South American camelids, electrical stimulation, meat aging, $\mathrm{pH}$, rigormortis, sensory characteristics

\section{Resumen}

El objetivo del presente trabajo fue demostrar que la estimulación eléctrica tiene un efecto significativo sobre la calidad de la carne de la alpaca en los aspectos de terneza, jugosidad, sabor y olor; y que además influye en la capacidad de su conservación. Se utilizó 12 alpacas hembras adultas de 4-4.5 años de edad y 50-60 kg p.v. Las canales fueron estimuladas eléctricamente a los 60 minutos del sacrificio, usando electrodos colocados en el tejido muscular. A cada media canal tratada se le estimuló con 500 voltios por 60 (T1) ó 30 segundos (T2), y con 600 voltios por 60 (T3) ó 30 segundos (T4); dejando sin estimulación eléctrica a las medias canales control. El tratamiento tuvo un efecto significativo $(\mathrm{p}=0.00)$ sobre el $\mathrm{pH}$ de la carne tratada, produciendo un menor crecimiento microbiano a las 24 horas post estimulación $(\mathrm{p}=0.004)$, especialmente en el tratamiento T2. En las características organolépticas se obtuvo un efecto significativo para el olor con el tratamiento $\mathrm{T} 1(\mathrm{p}=0.009)$, sabor con el tratamiento $\mathrm{T} 4(\mathrm{p}=0.002)$, terneza con los tratamientos $\mathrm{T} 2(\mathrm{p}=0.023)$ y T3 $(\mathrm{p}=0.004)$, y jugosidad con el tratamiento $\mathrm{T} 4(\mathrm{p}=0.005)$.

Palabras clave: camélidos sudamericanos, estimulación eléctrica, maduración de la carne, $\mathrm{pH}$, rigor mortis, características sensoriales

\footnotetext{
${ }^{1}$ Práctica privada. E-mail: kike_gem@hotmail.com

${ }^{2}$ Laboratorio de Salud Pública y Salud Ambiental, FMV-UNMSM

${ }^{3}$ Laboratorio de Medicina Veterinaria Preventiva, FMV-UNMSM
} 
El Perú es un país que presenta un marcado déficit en producción de proteínas de origen animal, que generalmente se subsana mediante la importación de carnes del exterior. La carne de camélidos es una alternativa interesante para cubrir la demanda de la zona andina del país por las características de su producción extensiva, que la hacen la más económica, y por representar la forma más eficiente de transformación de la energía de la pradera nativa en proteína animal (Ruiz de Castilla, 1994).

La carne de alpaca es a veces considerada desabrida y de poco valor; sin embargo, otros la consideran similar a la del ovino, y que cuando está molida no se puede diferenciar con la del bovino. También hay quienes señalan que se parece a la carne porcina cuando es tierna. Se dice que la carne de llama es semejante a la de cerdo y superior a la de ovino, siendo más tierna en animales capones que en enteros; sin embargo, estos aspectos son subjetivos, no existiendo apreciaciones objetivas (Vilca, 1991).

Los factores que determinan la calidad de la carne fresca, refrigerada, congelada o sometida a cualquier otro tratamiento, involucran consideraciones sobre su valor nutritivo, el estado higiénico sanitario, las características sensoriales u organolépticas y su utilidad para el procesamiento. Estos factores se pueden agrupar en: aquellos determinados antes del nacimiento del animal (genéticos), aquellos que intervienen durante la vida del animal (ambiente) y aquellos que afectan los procesos siguientes a su producción (tecnología cárnica). Estos últimos son los que tienen mayor incidencia en la conservacióndelacalideddel producto Quiroga $e t$ al., 2001).
Dentro de lo que es tecnología cárnica, se encuentra el método de estimulación eléctrica. La historia indica que los primeros estudios se hicieron en 1940 en los Estados Unidos y sin mayor éxito. Posteriormente, en 1970 se utilizó esta tecnología para evitar el encogimiento de la carne de cordero cuando era sometida a procesos de refrigeración; y en 1975 se desarrollaron algunos prototipos de estimuladores que fueron incorporándose en los mataderos americanos. Estos prototipos han sido mejorados y en la actualidad existen modelos que permiten trabajar hasta con 300 animales por hora (Falla, 2000). En aves se ha utilizado 450 voltios por 15 segundos (Craig et al, 1999; Sams, 1988) y 550 voltios en canales de toros Brahman (Solomón et al., 1986).

El presente trabajo tuvo por objetivo establecer si la estimulación eléctrica tiene un efecto significativo sobre la calidad de la carne de alpaca en sus aspectos de terneza, jugosidad, sabor y olor; así como evaluar su efecto en la capacidad de conservación.

\section{Lugar de estudio y animales}

Se llevó a cabo en el Laboratorio de Anatomía Animal y en el Laboratorio de Salud Pública y Salud Ambiental de la Facultad de Medicina Veterinaria de la Universidad Nacional Mayor de San Marcos, Lima, Perú.

Se utilizaron 12 alpacas hembras adultas de 4-4.5 años de edad y con pesos entre $50-60 \mathrm{~kg}$, provenientes de la sierra central del país y que permanecieron en Lima cerca de dos semanas antes del beneficio. En ese periodo fueron alimentadas con una mezcla de chala y alimento balanceado compuesto por harina de pescado, algarrobo, pepa de algodón, torta de soya, maíz, polvillo de arroz, aceites vegetales, melaza, pasta de algodón 
y subproductos de trigo (NDT: $64 \%$, proteína: $18 \%$ ). Cada animal recibió un promedio de concentrado de $2 \mathrm{~kg}$ por día.

\section{Sacrificio y procesamiento}

Los animales fueron sometidos a un faenado convencional, el cual se inició con un aturdimiento por conmoción, seguido de sangría, degüello, desollado y eviscerado. Las canales fueron divididas por la mitad y el proceso se terminó con el lavado y retoque pertinente (Vilca, 1991). Las canales tuvieron un periodo de oreo de 24 horas a la sombra y a una temperatura media de $15{ }^{\circ} \mathrm{C}$. Una mitad de la canal se destinó para tratamiento y la otra para control.

La mitad de las canales fueron estimuladas eléctricamente a los 60 minutos post sacrificio mediante electrodos colocados en el tejido muscular. Se hizo cuatro tipos de estimulaciones eléctricas: T1: 500 voltios por 60 segundos, T2: 500 voltios por 30 segundos, T3: 600 voltios por 60 segundos, y T4: 600 voltios por 30 segundos. Las medias canales del grupo control no recibieron estimulación eléctrica. Los voltajes y tiempos de estimulación eléctrica usados fueron determinados mediante un trabajo previo, llegándose a la conclusión que 500-600 voltios por 30-60 segundos eran los más indicados (M. Vilca, Comunicación Personal).

\section{Evaluaciones}

La medición del pH se hizo en el músculo Longissimus dorsi de todas las medias canales. Las lecturas se hicieron a la 1, 2, 3, $4,5,6,8,10,12,18$ y 24 horas post estimulación, usándose un potenciómetro digital portátil Cole Parmer, modelo 5985-80, con un electrodo y termómetro de inserción anexo para corrección automática del $\mathrm{pH}$.

La evaluación de la capacidad de conservación de la carne se realizó a través del número de microorganismos presentes antes de la estimulación eléctrica y a las 24 horas post estimulación. Las muestras fueron procesadas por el método Standar Plate
Count (SPC) que determina el número de microorganismos totales.

Para la evaluación organoléptica se contó con un panel de 13 jueces previamente capacitados y entrenados para efectuar el análisis sensorial. Se usaron fichas con escala hedónica para la evaluación sensorial de muestras tomadas del músculo Longissimus dorsi. Las muestras estuvieron almacenadas en congelación $\left(-18^{\circ} \mathrm{C}\right)$ por dos semanas. Se cocinaron por medio de calor seco (horneado a $80{ }^{\circ} \mathrm{C}$ por 10 minutos) en trozos de 20 a 25 $\mathrm{g}$, sin condimentos, envueltas en papel aluminio de uso alimentario.

\section{Analisis de datos}

Los datos relacionados al $\mathrm{pH}$ fueron analizados mediante regresión múltiple y análisis de varianza. Los resultados del estudio microbiológico se analizaron mediante el test de Kruskal Wallis. Los resultados de la evaluación organoléptica se analizaron por medio del test de Friedman (Gónzalez y Falcón, 1999).

En el Cuadro 1 se muestran los valores promedio del $\mathrm{pH}$ para cada uno de los tratamientos aplicados y del grupo control, en cada una de las horas post estimulación eléctrica consideradas en el diseño experimental.

Se encontró un efecto altamente significativo $(\mathrm{p}=0.00)$ en el $\mathrm{pH}$ de las carnes tratadas con la estimulación eléctrica vs. el grupo control. Esta diferencia se debe principalmente al voltaje aplicado que ocasiona un rápido descenso del $\mathrm{pH}$, especialmente en el grupo T2 $(500 \mathrm{v} / 30 \mathrm{~s})$, que provocó el mayor descenso (pH: 5.1) en el menor tiempo post estimulación ( 2 horas). Se pudo notar que, en general, las carnes estimuladas registran un $\mathrm{pH}$ menor a 6.0 durante las 24 primeras horas post estimulación, en contraposición al grupo control, cuyo $\mathrm{pH}$ se mantiene en ?6. Este 
Cuadro 1. pH del músculo Longissimus dorsi a diferentes horas post estimulación eléctrica en canales de alpacas

\begin{tabular}{|c|c|c|c|c|c|}
\hline \multirow{2}{*}{ Horas } & \multicolumn{2}{|c|}{500 voltios } & \multicolumn{2}{|c|}{600 voltios } & \multirow{2}{*}{ Control } \\
\hline & $60 \mathrm{seg}^{1}$ & $30 \mathrm{seg}$ & $60 \mathrm{seg}$ & $30 \mathrm{seg}$ & \\
\hline 1 & 5.85 & 5.38 & 5.85 & 5.32 & 6.38 \\
\hline 2 & 5.86 & 5.11 & 5.53 & 5.56 & 6.06 \\
\hline 3 & 5.82 & 5.75 & 5.36 & 5.74 & 5.89 \\
\hline 4 & 5.53 & 5.69 & 5.59 & 5.93 & 6.10 \\
\hline 5 & 5.52 & 5.78 & 5.35 & 6.14 & 6.00 \\
\hline 6 & 5.86 & 6.07 & 5.87 & 5.94 & 6.06 \\
\hline 8 & 5.58 & 5.61 & 5.17 & 5.62 & 5.62 \\
\hline 10 & 5.82 & 5.65 & 5.67 & 5.62 & 6.10 \\
\hline 12 & 5.68 & 5.61 & 5.8 & 5.48 & 6.52 \\
\hline 18 & 5.5 & 5.27 & 5.45 & 5.4 & 6.04 \\
\hline 24 & 5.42 & 5.27 & 5.65 & 5.31 & 6.31 \\
\hline
\end{tabular}

${ }^{1}$ Segundos

Cuadro 2. Cargas y tasas de crecimiento microbiano de la carne $e^{1}$ de alpacas entre los 60 minutos del sacrificio y las 24 horas de la estimulación eléctrica

\begin{tabular}{|c|c|c|c|}
\hline \multirow{2}{*}{ Tratamiento $^{2}$} & \multicolumn{2}{|c|}{ Carga microbiana $^{3}(\mathrm{ufc} / \mathrm{g})^{4}$} & \multirow{2}{*}{$\begin{array}{l}\text { Tasa de crecimiento } \\
\text { (\%) }\end{array}$} \\
\hline & $? 1$ hora & 24 horas & \\
\hline $\mathrm{T} 1$ & 16.67 & $14.2^{\mathrm{c}}$ & $12.7 \mathrm{c}$ \\
\hline $\mathrm{T} 2$ & 11.50 & $3.0^{\mathrm{a}}$ & $8.7 \mathrm{~b}$ \\
\hline $\mathrm{T} 3$ & 19.67 & $10.0^{\mathrm{b}}$ & $5.7 \mathrm{a}$ \\
\hline $\mathrm{T} 4$ & 4.50 & $4.2^{\mathrm{a}}$ & $11.7 \mathrm{c}$ \\
\hline Control & 11.92 & $17.2^{\mathrm{d}}$ & $15.3 \mathrm{~d}$ \\
\hline
\end{tabular}

${ }^{1}$ Músculo Longissimus dorsi

${ }^{2} \mathrm{~T} 1$ y T2: $500 \mathrm{v}$ por 60 y $30 \mathrm{seg}$, respectivamente; T3 y T4: $600 \mathrm{v}$ por 60 y 30 seg, respectivamente

3 muestras por tratamiento

${ }^{4}$ Unidades Formadoras de Colonias por gramo de carne

$a, b, c, d$ Letras distintas dentro de columnas indican diferencias significativas ( $p ? 0.05)$ 
Cuadro 3. Evaluación sensorial organoléptica del olor, sabor, terneza y jugosidad de carne de alpaca sometida a estimulación eléctrica (rango de 0 a $10^{1}$ )

\begin{tabular}{ccccc}
\hline Grupo $^{2}$ & Olor & Sabor & Terneza & Jugosidad \\
\hline T1 & 1.29 & 1.35 & 1.40 & 1.47 \\
C1 & 1.71 & 1.65 & 1.60 & 1.53 \\
Signif. $^{3}$ & $0.009^{*}$ & 0.05 & 0.19 & 0.74 \\
T2 & 1.53 & 1.53 & 1.32 & 1.55 \\
C2 & 1.47 & 1.47 & 1.68 & 1.45 \\
Signif. & 0.75 & 0.75 & $0.02^{*}$ & 0.51 \\
T3 & 1.35 & 1.47 & 1.27 & 1.60 \\
C3 & 1.65 & 1.53 & 1.73 & 1.40 \\
Signif. & 0.05 & 0.74 & $0.004^{*}$ & 0.18 \\
T4 & 1.50 & 1.74 & 1.36 & 1.72 \\
C4 & 1.50 & 1.26 & 1.64 & 1.28 \\
Signif. & 1.00 & $0.002^{*}$ & 0.08 & $0.005^{*}$ \\
\hline
\end{tabular}

${ }_{1}^{*}$ Resultados estadísticamente significativos

${ }^{1}$ Olor de inodoro a intenso; sabor de insípido a sabroso; terneza de blando a duro; jugosidad de seco a jugoso

${ }^{2}$ T1 y T2: $500 \mathrm{v}$ por 60 y $30 \mathrm{seg}$, respectivamente; T3 y T4: $600 \mathrm{v}$ por 60 y 30 seg, respectivamente.

C1 a C4: Grupo control (Medias canales de las que fueron tratadas)

${ }^{3}$ Nivel de significancia estadística mediante el test de Friedman

fenómeno pudo deberse a que la estimulación eléctrica incrementó el ciclo de contracción en los músculos, lo cual acelera el agotamiento del glucógeno de una manera más rápida y por lo tanto, ocasiona una mayor producción de ácido láctico (Hearnshaw, 1999). Quiroga et al. (2001) mencionan que el $\mathrm{pH}$ puede seguir descendiendo hasta 5.3 ó hasta que las reservas de glucógeno se agoten totalmente.

La estimulación eléctrica ocasiona un menor crecimiento microbiano a las 24 horas post estimulación en todos los tratamientos con relación al grupo control $(\mathrm{p}=0.004)$. El T2 $(500 \mathrm{v} / 30 \mathrm{~s})$ fue el de menor crecimiento microbiano seguidamente del T4 (600v/30s). Asimismo, el T3 fue el que obtuvo la menor tasa de crecimiento seguido del T2. El menor crecimiento microbiano se debió al ácido láctico que crea un medio desfavorable para el desarrollo de microorganismos (Téllez, 1992).
Se sabe que el olor de los alimentos se debe a la impresión sobre la superficie olfatoria que dejan los elementos volátiles que contienen los alimentos. Al evaluar los datos obtenidos en el presente trabajo se pudo observar que el tratamiento $\mathrm{T} 1(500 \mathrm{v} / 60 \mathrm{~s})$ fue el único que atenuó en forma significativa el olor de la carne (Cuadro 3).

El sabor sólo pudo ser mejorado con el tratamiento T4 $(600 \mathrm{v} / 30 \mathrm{~s})$. Es posible que a este nivel de voltaje y tiempo de estimulación se haya logrado obtener una mejor movilización de los aminoácidos libres de la carne, tal como lo menciona Sekikawa et al. (1999).

La terneza de la carne, el elemento de mayor importancia para el consumidor, mejoró significativamente con los tratamientos T2 (500v/30s) y T3 (600v/60s), aunque se puede decir que en los otros dos tratamientos se observó una tendencia de la carne hacia 
la blandura. Esta mejora se pudo deber a que la estimulación eléctrica conlleva a una mayor o más rápida liberación de las enzimas proteolíticas, llegando a una desnaturalización temprana de los haces musculares (Lawrie, 1998).

La jugosidad se mejoró únicamente con el tratamiento T4 (600v/30s). Se sabe que la jugosidad contiene muchos de los componentes del sabor y que además facilitan la fragmentación y ablandamiento de la carne durante el proceso de la masticación (Huerta Leindez, 2002).

La estimulación eléctrica:

? Produce una disminución rápida del $\mathrm{pH}$ post mortem.

? Ocasiona un menor crecimiento microbiano a las 24 horas post estimulación, con lo que se consigue una mejora en la condición higiénico sanitaria de la carne.

? El olor, la jugosidad y la terneza se mejoran significativamente dependiendo del voltaje y tiempo empleado en la estimulación.

1. Craig, E.; D. Fletcher; P. Papinaho. 1999. The effects of antemortem electrical stuning and postmortem electrical stimulation on biochemical and textural properties of broiler breast meat.
Poultry Sci. 78: 490-494.

2. Falla, L. 2000. La estimulación de la carne en canal de bovino. Carne 13: 19-22.

3. González, A.; N. Falcón. 1999. Análisis de datos en Medicina Veterinaria. Pub. Tec. $\mathrm{N}^{\circ}$ 49. Facultad de Medicina Veterinaria, UNMSM. Lima.

4. Hearnshaw, H. 1999. Como mejorar la calidad comestible de la carne de res. $1^{\text {ra }}$ parte. Asociación Colombiana de Criadores de Ganado Cebú. El Cebú 311: 54-60.

5. Huerta-Leidenz, N. 2002. Caracterización de ganado y carne bovina como base científica de la clasificación de canales en el trópico americano. En: Memorias XI Congreso Venezolano de Producción e Industria Animal. ULA-Venezuela. p 1-18.

6. Lawrie, R. 1998. Ciencia de la carne. $3^{\text {ra }}$ ed. p 105-113. Ed. Acribia. Zaragoza.

7. Quiroga, G.; J. García de Salas; J. López. 2001. Manual para el Curso-Taller: Tecnología de carnes y productos cárnicos. FAO. p 8-12.

8. Ruizde Castilla, M. 1994 Camelicultura: alpacas y llamas del sur del Perú. p 164174. Ed. Mercantil. Cusco.

9. Sekikawa, M.; K. Seno; K. Shimada; M. Fukushima; M. Mikami. 1999. Transaminase affects accumulation of free amino acids in electrically stimulated beef. J. Food Sci. 64: 384-386.

10. Sams, A. 1999. Meat quality during processing. Poult Sci. 78: 798-803.

11. Solomón, M.; R. West; J. Hentges. 1986. Effects of slaughter weight and carcass electrical stimulation on the quality and palatability of beef from young purebred bulls. J. Anim Sci. 63: 1838-1844.

12. Téllez, J. 1992. Tecnología e industrias cárnicas. Tomo I. p 162-167. Ed. Acribia. Zaragoza.

13. Vilca, M. 1991. Producción, tecnología e higiene de la carne. En: Avances y perspectivas del conocimiento de los camélidos sudamericanos. FAO, Chile. p 387- 417. 